Pacific Journal of Mathematics

SOME THEOREMS ON GENERALIZED

CARLITZ 


\section{SOME THEOREMS ON GENERALIZED DEDEKIND-RADEMACHER SUMS}

\section{Carlitz}

Radamacher has defined a generalized Dedekind sum

$$
s(h, k ; x, y)=\sum_{a(\bmod k)}\left(\left(h \frac{a+y}{k}+x\right)\right)\left(\left(\frac{a+y}{k}\right)\right)
$$

and proved a reciprocity theorem for this sum that generalizes the well known result for $s(h, k)$. In the present paper we define

$$
\begin{aligned}
& \phi_{r, s}(h, k ; x, y)=\sum_{a(\bmod k)} \bar{B}_{r}\left(h\left(\frac{a+y}{k}\right)+x\right) \bar{B}_{s}\left(\frac{a+y}{k}\right), \\
& \psi_{r, s}(h, k ; x, y)=\sum_{j=0}^{r}(-1)^{r-J}\left(\begin{array}{l}
r \\
j
\end{array}\right) h^{r-j} \phi_{j, r+s-J}(h, k ; x, y),
\end{aligned}
$$

where $\bar{B}_{n}(x)$ is the Bernoulli function, and show that

$$
\begin{aligned}
& (s+1) k^{s} \psi_{r+1, s}(h, k ; x, y)-(r+1) h^{r} \psi_{s+1, r}(k, h ; y, x) \\
& \quad=(s+1) k \bar{B}_{r+1}(x) \bar{B}_{s}(y)-(r+1) h \bar{B}_{r}(x) \bar{B}_{s+1}(y) \quad((h, k)=1) .
\end{aligned}
$$

We also prove the polynomial reciprocity theorem

$$
\begin{array}{r}
(1-v) \sum_{a=0}^{k-1} u^{h-[(h a+z) / k]} v^{a}-(1-u) \sum_{b=0}^{h-1} v^{k-[(k b+z) / h]} u^{b}=u^{h}-v^{k} \\
((h, k)=1)
\end{array}
$$

as well as some related results.

1. Introduction. For real $x$ put

$$
((x))= \begin{cases}x-[x]-\frac{1}{2} & (x \neq \text { integer }) \\ 0 & (x=\text { integer })\end{cases}
$$

where $[x]$ denotes the greatest integer $\leqq x$. The Dedekind sum $s(h, k)$ is defined by 


$$
s(h, k)=\sum_{a(\bmod k)}\left(\left(\frac{a}{k}\right)\right)\left(\left(\frac{h a}{k}\right)\right) .
$$

The most striking property of $s(h, k)$ is the reciprocity theorem

$$
12 h k\{s(h, k)+s(k, h)\}=h^{2}-3 h k+k^{2}+1 \quad((h, k)=1) .
$$

For an excellent introduction and many references to Dedekind sums see [9].

The Bernoulli function $\bar{B}_{n}(x)$ is defined by

$$
\bar{B}_{n}(x)=B_{n}(x-[x]),
$$

where $B_{n}(x)$ is the Bernoulli polynomial defined by

$$
\frac{z e^{x z}}{e^{z}-1}=\sum_{n=0}^{\infty} B_{n}(x) \frac{z^{n}}{n !}
$$

Note that, for $x \neq$ integer, $\bar{B}_{1}(x)=((x))$.

Apostol [1], [2] defined the generalized sum

$$
s_{n}(h, k)=\sum_{r(\bmod k)} \bar{B}_{1}\left(\frac{r}{k}\right) \bar{B}_{n}\left(\frac{h r}{k}\right)
$$

and proved the reciprocity theorem

$$
\begin{array}{r}
(n+1)\left\{h k^{n} s_{n}(h, k)+k h^{n} s_{n}(k, h)\right\}=(B k+B h)^{n+1}+n B_{n+1} \\
\quad((h, k)=1) .
\end{array}
$$

This result is indeed valid for all $n \geqq 0$. For a simple proof see [4, §3].

A further generalization of (1.4) is furnished by

$$
\phi_{r, s}(h, k)=\sum_{a(\bmod k)} \bar{B}_{r}\left(\frac{a}{k}\right) \bar{B}_{s}\left(\frac{h a}{k}\right)
$$

where $r, s$ are arbitrary nonnegative integers. Put

$$
\psi_{r, s}(h, k)=\sum_{i=0}^{r}(-1)^{t}\left(\begin{array}{l}
r \\
t
\end{array}\right) h^{\prime} \phi_{r-t, s+t}(h, k) .
$$

The writer [3], [7] has proved the following reciprocity theorem which includes (1.5) as a special case. 
(1.8)

$$
\begin{aligned}
&(s+1) k^{s} \psi_{r+1, s}(h, k)-(s+1) k B_{r+1} B_{s}=(r+1) h^{r} \psi_{s+1, r}(k, h) \\
&-(r+1) h B_{s+1} B_{r} \quad((h, k)=1) .
\end{aligned}
$$

He has also proved the following polynomial reciprocity:

$$
\begin{gathered}
(u-1) \sum_{r=1}^{k-1} u^{k-r-1} v^{[h r / k]}-(v-1) \sum_{r=1}^{h-1} v^{h-r-1} u^{[k r / h]} \\
=u^{k-1}-v^{h-1} \quad((h, k)=1)
\end{gathered}
$$

where $u, v$ are indeterminates.

Rademacher [10] has generalized $s(h, k)$ in the following way:

$$
s(h, k ; x, y)=\sum_{a(\bmod k)}\left(\left(h \frac{a+y}{k}+x\right)\right)\left(\left(\frac{a+y}{k}\right)\right),
$$

where $x, y$ are arbitrary real numbers. He proved that

$$
\begin{aligned}
s(h, k ; x, y)+ & s(k, h ; y, x) \\
= & -\frac{1}{4} \delta(x) \delta(y)+((x))((y)) \\
& +\frac{1}{2}\left\{\frac{h}{k} \bar{B}_{2}(y)+\frac{1}{h k} \bar{B}_{2}(h y+k x)+\frac{k}{h} \bar{B}_{2}(x)\right\},
\end{aligned}
$$

where $(h, k)=1$ and

$$
\delta(x)= \begin{cases}1 & (x=\text { integer }) \\ 0 & (x \neq \text { integer })\end{cases}
$$

For a simplified version of the proof see [5].

In the present paper we define

$$
\phi_{r, s}(h, k ; x, y)=\sum_{a(\bmod k)} \bar{B}_{r}\left(h \frac{a+y}{k}+x\right) \bar{B}_{s}\left(\frac{a+y}{k}\right)
$$

and

$$
\psi_{r, s}(h, k ; x, y)=\sum_{j=0}^{r}(-1)^{r-1}\left(\begin{array}{l}
r \\
j
\end{array}\right) h^{r-J} \phi_{,, r+s-j}(h, k ; x, y)
$$

corresponding to (1.6) and (1.7), respectively. We prove the reciprocity theorem 
$(s+1) k^{s} \psi_{r+1, s}(h, k ; x, y)-(r+1) h^{r} \psi_{s+1, r}(k, h ; y, x)=(s+1) k \bar{B}_{r+1}(x) \bar{B}_{s}(y)$

$$
-(r+1) h \bar{B}_{r}(x) \bar{B}_{s+1}(y) \quad((h, k)=1) .
$$

It should be observed that there is no loss in generality in assuming that

$$
0 \leqq x<1, \quad 0 \leqq y<1
$$

We show also, assuming (1.15), that

$$
\begin{array}{r}
(1-v) \sum_{a=0}^{k-1} u^{h-[(h a+z) / k]} v^{a}-(1-u) \sum_{b=0}^{h-1} v^{k-[(k b+z) / h]} u^{b}=u^{h}-v^{k} \\
((h, k)=1),
\end{array}
$$

where $z=k x+h y$. For $x=y=0,(1.16)$ reduces to (1.9) after a little manipulation. Clearly (1.16) holds for all $z$ such that $0 \leqq z<h+k$.

For some additional results see $\$ 4$ below, in particular (4.1), (4.2), (4.3), (4.4), (4.5), (4.6).

2. Proof of (1.14). We recall that $[8, \mathrm{Ch} .2]$

$$
\bar{B}_{n}(h x)=h^{n-1} \sum_{b(\bmod h)} \bar{B}_{n}\left(x+\frac{b}{h}\right)
$$

Thus (1.12) becomes

$$
\phi_{r, s}(h, k ; x, y)=h^{r-1} \sum_{\substack{a(\bmod k) \\ b(\bmod h)}} \bar{B}_{r}\left(\frac{a+y}{k}+\frac{b+x}{h}\right) \bar{B}_{s}\left(\frac{a+y}{h}\right) .
$$

We shall write this in the abbreviated form

$$
\phi_{r, s}(h, k ; x, y)=h^{r-1} \sum_{a, b} \bar{B}_{r}(\alpha+\beta) \bar{B}_{s}(\alpha)
$$

where

$$
\alpha=\frac{a+y}{k}, \quad \beta=\frac{b+x}{h}
$$

and the summation on the right of (2.2) is over complete residue systems $(\bmod k)$ and $(\bmod h)$, respectively. 
Substituting from (2.2) in (1.13), we get

$$
\psi_{r, s}(h, k ; x, y)=h^{r-1} \sum_{j=0}^{r}(-1)^{r-1}\left(\begin{array}{l}
r \\
j
\end{array}\right) \sum_{a, b} \bar{B}_{l}(\alpha+\beta) \bar{B}_{r+s-j}(\alpha) .
$$

Now consider

$$
\begin{aligned}
& \Phi(h, k ; x, y ; u, v) \\
& \quad=\sum_{r, s=0}^{\infty} s k^{s-1} \psi_{r, s-1}(h, k ; x, y) \frac{u^{r} v^{s}}{r ! s !} \\
& \quad=\sum_{r, s} \frac{h^{r-1} k^{s-1} u^{r} v^{s}}{r !(s-1) !} \sum_{j=0}^{r}(-1)^{r-1}\left(\begin{array}{l}
r \\
j
\end{array}\right) \sum_{a=0}^{k-1} \sum_{b=0}^{h-1} \bar{B}_{j}(\alpha+\beta) \bar{B}_{r+s-\jmath-1}(\alpha) .
\end{aligned}
$$

We assume in what follows that

$$
0 \leqq x<1, \quad 0 \leqq y<1
$$

which implies

$$
0 \leqq \alpha<1, \quad 0 \leqq \beta<1
$$

Thus

$$
\bar{B}_{r+s+j-1}(\alpha)=B_{r+s-j-1}(\alpha)
$$

Taking $m=r+s-j-1,(2.5)$ becomes

$$
\begin{aligned}
& \Phi(h, k ; x, y ; u, v) \\
& =h^{-1} v \sum_{a=0}^{k-1} \sum_{b=0}^{h-1} \sum_{j=0}^{\infty} \frac{(h u)^{\gamma}}{j !} \bar{B}_{j}(\alpha+\beta) \sum_{m=0}^{\infty} \frac{1}{m !} B_{m}(\alpha) \\
& =h^{-1} v \sum_{a=0}^{m+1} \sum_{b=0}^{h-1} \sum_{j=0}^{\infty} \frac{(h u)^{y}}{j !} \bar{B}_{j}(\alpha+\beta) \sum_{m=0}^{\infty} \frac{(-h u+k v)^{m}}{m !} B_{m}(\alpha) \\
& =h^{-1} v \sum_{j, m=0}^{\infty} \frac{(h u)^{\prime}(-h u+k v)^{m}}{j ! m !} \sum_{a, b} \bar{B}_{j}(\alpha+\beta) B_{m}(\alpha) .
\end{aligned}
$$

Since

$$
B_{j}(x+1)-B_{j}(x)=j x^{\prime-1},
$$


the inner sum

$$
\begin{aligned}
& \sum_{a=0}^{k-1} \sum_{b=0}^{h-1} \bar{B}_{l}(\alpha+\beta) B_{m}(\alpha) \\
& \quad=\sum_{a, b} B_{l}(\alpha+\beta-[\alpha+\beta]) B_{m}(\alpha) \\
& \quad=\sum_{a, b} B_{l}(\alpha+\beta) B_{m}(\alpha)-\sum_{\substack{a, b \\
\alpha+\beta \geq 1}}\left(B_{l}(\alpha+\beta)-B_{l}(\alpha+\beta-1)\right) B_{m}(\alpha) \\
& \quad=\sum_{a, b} B_{l}(\alpha+\beta) B_{m}(\alpha)-j \sum_{\substack{a, b \\
\alpha+\beta \geq 1}}(\alpha+\beta-1)^{-1} B_{m}(\alpha) .
\end{aligned}
$$

Thus (2.8) becomes

(2.9) $\Phi(h, k ; x, y ; u, v)=\Phi_{1}(h, k ; x, y ; u, v)-\Phi_{2}(h, k ; x, y ; u, v)$,

where

$$
\begin{aligned}
\Phi_{1}(h, k ; x, y ; u, v)= & h^{-1} v \sum_{h, m=0}^{\infty} \frac{(h u)^{y}(-h u+k v)^{m}}{j ! m !} \\
& \cdot \sum_{a, b} B_{l}(\alpha+\beta) B_{m}(\alpha), \\
\Phi_{2}(h, k ; x, y ; u, v)= & h^{-1} v \sum_{,, m=0}^{\infty} \frac{(h u)^{y}(-h u+k v)^{m}}{j ! m !} \\
& \cdot \sum_{\substack{a, b \\
\alpha+\beta \geq 1}}(\alpha+\beta-1)^{-1} B_{m}(\alpha) .
\end{aligned}
$$

Clearly, by (1.3) and (2.3),

$$
\begin{aligned}
\Phi_{1}(h, k ; x, y ; u, v)= & h^{-1} v \frac{h u}{e^{h u}-1} \frac{-h u+k v}{e^{-h u+k v}-1} \\
& \cdot \sum_{a, b} e^{h u(\alpha+\beta)} e^{(-h u+k v) \alpha} \\
= & \frac{u v}{e^{h u}-1} \frac{-h u+k v}{e^{-h u+k v}-1} \cdot e^{x u+y v} \frac{e^{h u}-1}{e^{u}-1} \frac{e^{k v}-1}{e^{v}-1} \\
= & \frac{u v}{e^{u}-1} \frac{e^{k v}-1}{e^{v}-1} \frac{h u-k v}{e^{h u}-e^{k v}} e^{h u+x u+y v},
\end{aligned}
$$




$$
\begin{aligned}
\Phi_{2}(h, k ; x, y ; u, v) & =u v \frac{-h u+k v}{e^{-h u+k v}-1} \cdot \sum_{\substack{a, b \\
\alpha+\beta \geq 1}} e^{h u(\alpha+\beta-1)} e^{(-h u+k v) \alpha} \\
& =u v \frac{h u-k v}{e^{h u}-e^{k v}} e^{x u+y v} \sum_{\substack{a, b \\
\alpha+\beta \geqq 1}} e^{a v+b u} .
\end{aligned}
$$

It follows from (2.10) that

$$
\begin{aligned}
& \Phi_{1}(h, k ; x, y ; u, v)-\Phi_{1}(k, h ; y, x ; v, u) \\
& \quad=\frac{u}{e^{u}-1} \frac{v}{e^{v}-1} \frac{h u-k v}{e^{h u}-e^{k v}} e^{x u+y v}\left\{e^{h u}\left(e^{k v}-1\right)-e^{k v}\left(e^{h u}-1\right)\right\} \\
& \quad=(-h u+k v) \frac{u e^{x u}}{e^{u}-1} \frac{v e^{y v}}{e^{v}-1}
\end{aligned}
$$

while

$$
\Phi_{2}(h, k ; x, y ; u, v)-\Phi_{2}(k, h ; y, x ; v, u)=0 .
$$

Therefore, by (2.9), (2.12) and (2.13),

$$
\Phi(h, k ; x, y ; u, v)-\Phi(k, h ; y, x ; v, u)=(-h u+k v) \frac{u e^{x u}}{e^{u}-1} \frac{v e^{y v}}{e^{v}-1} .
$$

By (2.5), the left hand side of (2.14) is equal to

$$
\sum_{r, s=0}^{\infty}\left\{s k^{s-1} \psi_{r, s-1}(h, k ; x, y)-r h^{r-1} \psi_{s, r-1}(k, h ; y, x)\right\} \frac{u^{r} v^{s}}{r ! s !} .
$$

By (1.3), the right hand side of (2.14) is equal to

$$
\begin{aligned}
& (-h u+k v) \sum_{r, s=0}^{\infty} B_{r}(x) B_{s}(y) \frac{u^{r} v^{s}}{r ! s !} \\
& =\sum_{r, s=0}^{\infty}\left\{s k B_{r}(x) B_{s-1}(y)-r h B_{r-1}(x) B_{s}(y)\right\} \frac{u^{r} v^{s}}{r ! s !} .
\end{aligned}
$$

Hence, equating coefficients of $u^{r} v^{s} / r ! s !$, we get

$$
\begin{aligned}
s k^{s-1} \psi_{r, s-1}(h, k ; x, y)-r h^{r-1} \psi_{s, r-1}(k, h ; y, x) & =s k B_{r}(x) B_{s-1}(y) \\
& -r h B_{r-1}(x) B_{s}(y)
\end{aligned}
$$


Finally, dropping the restriction (2.6), we have

$$
\begin{aligned}
s k^{s-1} \psi_{r, s-1}(h, k ; x, y)-r h^{r-1} \psi_{s, r-1}(k, h ; y, x)= & s k \bar{B}_{r}(x) \bar{B}_{s-1}(y) \\
& -r h \bar{B}_{r-1}(x) \bar{B}_{s}(y)
\end{aligned}
$$

for all nonnegative $r, s$ and all real $x, y$.

3. Proof of (1.16). We again assume that

$$
0 \leqq x<1, \quad 0 \leqq y<1 .
$$

By (1.12) and (1.13) we have

$$
\psi_{r, s}(h, k ; x, y)=\sum_{j=0}^{r}(-1)^{r-1}\left(\begin{array}{l}
r \\
j
\end{array}\right) h^{r-1} \sum_{a=0}^{k-1} \bar{B}_{j}\left(h \frac{a+y}{k}+x\right) B_{r+s-\jmath}\left(\frac{a+y}{k}\right) .
$$

Then, as in the previous proof,

$$
\begin{aligned}
\Phi & (h, k ; x, y ; u, v) \\
& =\sum_{r, s=0}^{\infty} s k^{s-1} \psi_{r, s-1}(h, k ; x, y) \frac{u^{r} v^{s}}{r ! s !} \\
& =v \sum_{r, s=0}^{\infty} \frac{u^{r}(k v)^{s}}{r ! s !} \sum_{j=0}^{r}(-1)^{r-1}\left(\begin{array}{l}
r \\
j
\end{array}\right) h^{r-1} \sum_{a=0}^{k-1} \bar{B}_{l}\left(h \frac{a+y}{k}+x\right) B_{r+s-1}\left(\frac{a+y}{k}\right) \\
& =v \sum_{l, m=0}^{\infty} \frac{u^{\prime}(-h u+k v)^{m}}{j ! m !} \sum_{a=0}^{k-1} B_{l}\left(\frac{h a+z}{k}-\left[\frac{h a+z}{k}\right]\right) B_{m}\left(\frac{a+y}{k}\right) \\
& =v \frac{u}{e^{u}-1} \frac{-h u+k v}{e^{-h u+k v}-1} \sum_{a=0}^{k-1} \exp \left\{\left(\frac{h a+z}{k}-\left[\frac{h a+z}{k}\right]\right) u\right. \\
& =\frac{u v}{e^{u}-1} \frac{h u-k v}{e^{h u}-e^{k v}} e^{x u+y v} \sum_{a=0}^{k-1} \exp \left\{\left(h-\left[\frac{h a+z}{k}\right]\right) u+a v\right\},
\end{aligned}
$$

where

$$
z=k x+h y .
$$

It follows that 


$$
\begin{aligned}
& \Phi(h, k ; x, y ; u, v)-\Phi(k, h ; y, x ; v, u) \\
& =\frac{u v}{e^{u}-1} \frac{h u-k v}{e^{h u}-e^{k v}} e^{x u+y v} \sum_{a=0}^{k-1} \exp \left\{\left(h-\left[\frac{h a+z}{k}\right]\right) u+a v\right\} \\
& \quad-\frac{u v}{e^{v}-1} \frac{h u-k v}{e^{h u}-e^{k v}} e^{x u+y v} \sum_{b=0}^{h-1} \exp \left\{\left(k-\left[\frac{k b+z}{h}\right]\right) v+b u\right\}
\end{aligned}
$$

Comparing (3.3) with (2.14) and simplifying, we get

$$
\begin{aligned}
\left(e^{v}-1\right) & \sum_{a=0}^{k-1} \exp \left\{\left(h-\left[\frac{h a+z}{k}\right]\right) u+a v\right\} \\
& -\left(e^{u}-1\right) \sum_{b=0}^{h-1} \exp \left\{\left(k-\left[\frac{k b+z}{h}\right]\right) v+b u\right\}=-e^{h u}+e^{k v}
\end{aligned}
$$

Replacing $e^{u}, e^{v}$ by $u, v$, respectively, this becomes

$$
\begin{array}{r}
(1-v) \sum_{a=0}^{k-1} u^{h-[(h a+z) / k]} v^{a}-(1-u) \sum_{b=0}^{h-1} v^{k-[(k b+z) / h]} u^{b}=u^{h}-v^{k} \\
((h, k)=1) .
\end{array}
$$

Clearly (3.5) is a polynomial identity in the indeterminates $u, v$. It is not evident how the restriction (3.1) can be removed.

To show that (3.5) includes (1.9), take $x=y=z=0$ and replace $a$ by $k-a, b$ by $k-b$. Thus the left hand side of (3.5) becomes

$$
\begin{aligned}
(1-v) u^{h}-(1-u) v^{k} \\
\quad+(1-v) \sum_{a=1}^{k-1} u^{h-[h-(h a / k)]} v^{k-a}-(1-u) \sum_{b=1}^{h-1} v^{k-[k-(k b / h)]} u^{h-b} \\
=\left(u^{h}-v^{k}\right)-u v\left(u^{h-1}-v^{k-1}\right) \\
\quad+(1-v) \sum_{a=1}^{k=1} u^{[h a / k]+1} v^{k-a}-(1-u) \sum_{b=1}^{h-1} v^{[k b / h]+1} u^{h-b}
\end{aligned}
$$

since

$$
[m-x]=m-1-[x] \quad(m=\text { integer, } x \neq \text { integer }) .
$$

Thus we get 


$$
(1-v) \sum_{a=1}^{k-1} u^{[h a / k]} v^{k-a-1}-(1-u) \sum_{b=1}^{h-1} v^{[k b / h]} u^{h-b-1}=u^{h-1}-v^{k-1}
$$

which is (1.9) in a slightly different notation.

4. Additional results. We have

$$
\begin{aligned}
& \left(e^{v}-1\right) \sum_{a=0}^{k-1} \exp \left\{\left(h-\left[\frac{h a+z}{k}\right]\right) u+a v\right\} \\
& =\sum_{a=0}^{k-1} \sum_{r=0}^{\infty}\left(h-\left[\frac{h a+z}{k}\right]\right)^{r} \frac{u^{r}}{r !} \sum_{s=0}^{\infty}\left((a+1)^{s}-a^{s}\right) \frac{v^{s}}{s !}
\end{aligned}
$$

Thus the left hand side of (3.4) is equal to

$$
\begin{aligned}
\sum_{r, s=0}^{\infty} \frac{u^{r} v^{s}}{r ! s !}\left\{\sum_{a=0}^{k-1}\left(h-\left[\frac{h a+z}{k}\right]\right)^{r}\left((a+1)^{s}-a^{s}\right)\right. \\
\left.-\sum_{b=0}^{h-1}\left(k-\left[\frac{k b+z}{h}\right]\right)^{s}\left((b+1)^{r}-b^{r}\right)\right\}
\end{aligned}
$$

Since the right hand side of (3.4) is equal to

$$
-\sum_{r=0}^{\infty} \frac{h^{r} u^{r}}{r !}+\sum_{s=0}^{\infty} \frac{k^{s} v^{s}}{s !}
$$

we get

$$
\begin{array}{r}
\sum_{a=0}^{k-1}\left(h-\left[\frac{h a+z}{k}\right]\right)^{r}\left((a+1)^{s}-a^{s}\right)-\sum_{b=0}^{h-1}\left(k-\left[\frac{k b+z}{h}\right]\right)^{s}\left((b+1)^{r}-b^{\prime}\right) \\
=-h^{r} \delta_{s, 0}+k^{s} \delta_{r, 0}, \quad((h, k)=1)
\end{array}
$$

for all nonnegative $r, s$ and all $z$ such that

$$
0 \leqq z<h+k
$$

Hence, in particular,

$$
\sum_{a=0}^{k-1}\left(h-\left[\frac{h a+z}{k}\right]\right)^{\prime}\left((a+1)^{s}-a^{s}\right)=\sum_{b=0}^{h-1}\left(k-\left[\frac{k b+z}{h}\right]\right)^{s}\left((b+1)^{r}-b^{r}\right)
$$

$$
(r>0, s>0 ; 0 \leqq z<h+k)
$$


For example, for $r=s=2$,

$$
\begin{array}{r}
\sum_{a=0}^{k-1}(2 a+1)\left(h-\left[\frac{h a+z}{k}\right]\right)^{2}=\sum_{b=0}^{h-1}(2 b+1) \\
\left(k-\left[\frac{k b+z}{h}\right]\right)^{2} \\
(0 \leqq z<h+k) .
\end{array}
$$

For $s=1$ we get

$$
\begin{array}{r}
\sum_{a=0}^{-1}\left(h-\left[\frac{h a+z}{k}\right]\right)^{r}=\sum_{b=0}^{h-1}\left(k-\left[\frac{k b+z}{h}\right]\right)\left((b+1)^{r}-b^{r}\right) \\
(r>0, \quad 0 \leqq z<h+k) .
\end{array}
$$

Recall [8, Ch. 2] that

$$
\begin{aligned}
n x^{n-1} & =B_{n}(x+1)-B_{n}(x) \\
& =\sum_{j=0}^{n}\left(\begin{array}{l}
n \\
j
\end{array}\right) B_{n-1}\left((x+1)^{j}-x^{\prime}\right),
\end{aligned}
$$

where $B_{n}=B_{n}(0)$ is the $n$th Bernoulli number. Thus if in (4.1) we replace $r, s$ by $i, j$, respectively, multiply both sides by

$$
\left(\begin{array}{l}
r \\
i
\end{array}\right)\left(\begin{array}{l}
s \\
j
\end{array}\right) B_{r-i} B_{s-1}
$$

and sum over $i, j$, we get

$$
\begin{gathered}
s \sum_{a=0}^{k-1} a^{s-1} B_{r}\left(h-\left[\frac{h a+z}{k}\right]\right)-r \sum_{b=0}^{h-1} b^{r-1} B_{s}\left(k-\left[\frac{k b+z}{h}\right]\right) \\
=B_{s}(k) B_{r}-B_{r}(h) B_{s} \quad(0 \leqq z<h+k) .
\end{gathered}
$$

A more general result is

$$
\begin{array}{r}
s \sum_{a=0}^{k-1}(a+\eta)^{s-1} B_{r}\left(h+\xi-\left[\frac{h a+z}{k}\right]\right) \\
-r \sum_{b=0}^{h-1}(b+\xi)^{r-1} B_{s}\left(k+\eta-\left[\frac{k b+z}{h}\right]\right) \\
=B_{r}(\xi) B_{s}(k+\eta)-B_{r}(h+\xi) B_{s}(\eta) \quad(0 \leqq z<h+k),
\end{array}
$$

where $\xi$ and $\eta$ are arbitrary. In particular, for $\xi=1-h, \eta=1-k,(4.5)$ 
reduces to

$$
\begin{gathered}
s \sum_{a=0}^{k-1}(a+1-k)^{s-1} B_{r}\left(1-\left[\frac{h a+z}{k}\right]\right)-r \sum_{b=0}^{h-1}(b+1-h)^{r-1} B_{s}\left(1-\left[\frac{k b+z}{h}\right]\right) \\
=B_{r}(1-h) B_{s}(1)-B_{r}(1) B_{s}(1-k) .
\end{gathered}
$$

Since

$$
B_{n}(1-x)=(-1)^{n} B_{n}(x)
$$

we get

$$
\begin{aligned}
& s \sum_{a=0}^{k-1}(k-a-1)^{s-1} B_{r}\left(\left[\frac{h a+z}{k}\right]\right)-r \sum_{b=0}^{h-1}(h-b-1)^{r-1} B_{s}\left(\left[\frac{k b+z}{h}\right]\right) \\
& =-B_{r}(h) B_{s}+B_{r} B_{s}(k) \quad(0 \leqq z<h+k) .
\end{aligned}
$$

\section{REFERENCES}

1. T. M. Apostol, Generalized Dedekind sums, Duke Math. J., 17 (1950), 147-157.

2. —- Theorems on generalized Dedekind sums, Pacific J. Math., 2 (1952) 1-9.

3. L. Carlitz, A reciprocity and four-term relation for generalized Dedekind sums, Indagationes Mathematicae, 36 (1974), 413-422.

4. __ The reciprocity theorem for Dedekind sums, Pacific J. Math., 3 (1953), 523-527.

5. — The reciprocity theorem for Dedekind-Rademacher sums, Acta Arithmetica, 29 (1975), 309-313.

6. - Some polynomials associated with Dedekind sums, Acta Mathematica Scientarium Hungaricae, 26 (1975), 311-319.

7. _- Some theorems on generalized Dedekind sums, Pacific J. Math., 3 (1953), 513-522.

8. N. E. Nörlund, Vorlesungen über Differenzenrechnung, Springer, Berlin, 1924.

9. H. Rademacher and E. Grosswald, Dedekind Sums, Mathematical Association of America, Washington, D.C., 1972.

10. H. Rademacher, Some remarks on certain generalized Dedekind sums, Acta Arithmetica, 9 (1964), 97-105.

Received September 13, 1976

DUKe UnIVERSTrY

DURHAM, NC 27706 


\section{PACIFIC JOURNAL OF MATHEMATICS}

\section{EDITORS}

RICHARD ARENS (Managing Editor)

University of California

Los Angeles, CA 90024

R. A. BeAumont

University of Washington

Seattle, WA 98105

C. C. MOORE

University of California

Berkeley, CA 94720
J. DUGUNDJI

Department of Mathematics

University of Southern California

Los Angeles, CA 90007

R. FINN AND J. MILGRAM

Stanford University

Stanford, CA 94305

\section{ASSOCIATE EDITORS}
E. F. BECKENBACH
B. H. NEUMANN
F. WOLF
K. YoshidA

\section{SUPPORTING INSTITUTIONS}

UNIVERSITY OF BRITISH COLUMBIA

UNIVERSITY OF SOUTHERN CALIFORNIA

CALIFORNIA INSTITUTE OF TECHNOLOGY

STANFORD UNIVERSITY

UNIVERSITY OF CALIFORNIA

UNIVERSITY OF HAWAII

MONTANA STATE UNIVERSITY

UNIVERSITY OF TOKYO

UNIVERSITY OF NEVADA

UNIVERSITY OF UTAH

NEW MEXICO STATE UNIVERSITY

OREGON STATE UNIVERSITY

UNIVERSITY OF OREGON

OSAKA UNIVERSITY

WASHINGTON STATE UNIVERSITY

UNIVERSITY OF WASHINGTON

AMERICAN MATHEMATICAL SOCIETY

The Supporting Institutions listed above contribute to the cost of publication of this Journal, but they are not owners or publishers and have no responsibility for its contents or policies.

Mathematical papers intended for publication in the Pacific Journal of Mathematics should be in typed form or offset-reproduced (not dittoed), double spaced with large margins. Underline Greek letters in red, German in green, and script in blue. The first paragraph or two must be capable of being used separately as a synopsis of the entire paper. Items of the bibliography should not be cited there unless absolutely necessary, in which case they must be identified by author and Journal, rather than by item number. Manuscripts, in duplicate, may be sent to any one of the four editors. Please classify according to the scheme of Math. Reviews, Index to Vol. 39. All other communications should be addressed to the managing editor, or Elaine Barth, University of California, Los Angeles, California, 90024.

100 reprints are provided free for each article, only if page charges have been substantially paid. Additional copies may be obtained at cost in multiples of 50 .

The Pacific Journal of Mathematics is issued monthly as of January 1966. Regular subscription rate: $\$ 72.00$ a year (6 Vols., 12 issues). Special rate: $\$ 36.00$ a year to individual members of supporting institutions.

Subscriptions, orders for numbers issued in the last three calendar years, and changes of address should be sent to Pacific Journal of Mathematics, 103 Highland Boulevard, Berkeley, California, 94708.

PUBLISHED BY PACIFIC JOURNAL OF MATHEMATICS, A NON-PROFIT CORPORATION

Printed at Jerusalem Academic Press, POB 2390, Jerusalem, Israel. 


\section{Pacific Journal of Mathematics}

\section{Vol. 75, No. $2 \quad$ October, 1978}

Susan Jane Zimmerman Andima and W. J. Thron, Order-induced

topological properties ................................... 297

Gregory Wade Bell, Cohomology of degree 1 and 2 of the Suzuki groups . . 319

Richard Body and Roy Rene Douglas, Rational homotopy and unique

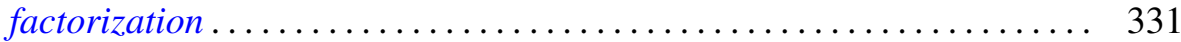

Frank Lewis Capobianco, Fixed sets of involutions ................. 339

L. Carlitz, Some theorems on generalized Dedekind-Rademacher sums .... 347

Mary Rodriguez Embry and Alan Leslie Lambert, The structure of a special class of weighted translation semigroups .....................

Steve Ferry, Strongly regular mappings with compact ANR fibers are

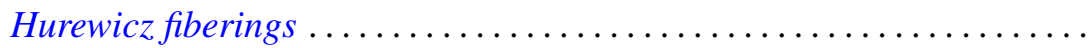

Ivan Filippenko and Marvin David Marcus, On the unitary invariance of the

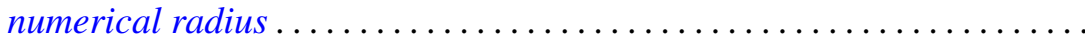

$\mathrm{H}$. Groemer, On the extension of additive functionals on classes of convex

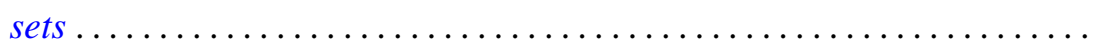

Rita Hall, On the cohomology of Kuga's fiber variety ............... 411

H. B. Hamilton, Congruences on $\mathrm{N}$-semigroups ................. 423

Manfred Herrmann and Rolf Schmidt, Regular sequences and lifting

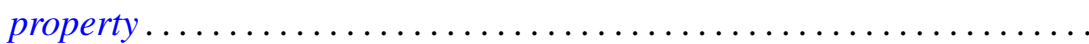

James Edgar Keesling, Decompositions of the Stone-Čech compactification which are shape equivalences .....................

Michael Jay Klass and Lawrence Edward Myers, On stopping rules and the expected supremum of $S_{n} / T_{n}$

Ronald Charles Linton, $\lambda$-large subgroups of $C_{\lambda}$-groups

William Owen Murray, IV and L. Bruce Treybig, Triangulations with the free cell property ............................

Louis Jackson Ratliff, Jr., Polynomial rings and $H_{i}$-local rings ...

Michael Rich, On alternate rings and their attached Jordan rings....

Gary Sampson and H. Tuy, Fourier transforms and their Lipschitz

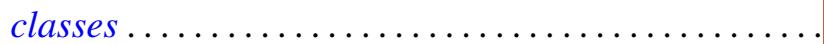

Helga Schirmer, Effluent and noneffluent fixed points on dendrites ...

Daniel Byron Shapiro, Intersections of the space of skew-symmetric maps

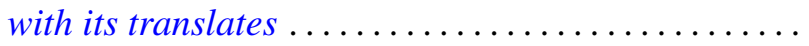

Edwin Spanier, Tautness for Alexander-Spanier cohomology ...

Alan Stein and Ivan Ernest Stux, A mean value theorem for binary digits ...

Franklin D. Tall, Normal subspaces of the density topology . .

William Yslas Vélez, Prime ideal decomposition in $F\left(\mu^{1 / p}\right) \ldots$ 